\title{
Survey of MAC Layer Protocols for vehicular Ad
}

\section{Hoc Network}

\author{
Vimal Bibhu \\ Research Scholar, Department of IT, B.R.A Bihar University, Mujaffarpur, Bihar, India \\ Email:vimalbibhu@gmail.com \\ Dr. Dhirendra Kumar Singh, \\ University Department of Mathematics, B.R.A Bihar University, Mujaffarpur, Bihar, India
}

\begin{abstract}
Vehicular Ad Hoc Network is currently challenge for the wireless networking and its researchers. In this paper we have proposed the survey of the different MAC Layer protocols of the wireless medium those can be implemented in the Vehicular Ad Hoc Networking. The survey is based upon the study of the different protocol on their MAC level and its performance factors is evaluated. The performance factor is extracted from the studied materials and current working conditions of the protocols. These all factors are mobility, accuracy, privacy and confidentiality, safety critical message priority, delay control and suitability on vehicle and roadside. According to analyzed performance factor the IEEE 802.11p Wave is most suitable protocol for the Vehicular Ad Hoc Network but it does not cover up all conditional requirements.
\end{abstract}

Index Terms- Intelligent Transportation service, InterVehicular Communication, Medium Access Control, Distributed Coordination Function.

\section{Introduction}

The improvement of the network technologies and infrastructure have given the opportunity to use it in several different fields. One of the most emergent field of applications of ad hoc network technology is the development of the Vehicular Ad-hoc Networks (VANETs). There are two different kinds of communications such as Vehicle to Vehicle(V2V) and Vehicle to Roadside(V2R) under the Vehicular Ad Hoc Networking. VANETs are developed for a set of communicating vehicles with sensors and roadside wireless devices to provide interconnection among each other without any pre-existing infrastructure (ad-hoc mode). The most important network technology available nowadays for establishing VANETs is the IEEE 802.11b (Wi-Fi) standard, nevertheless new standards as IEEE 802.11p or IEEE 802.16 (WiMax) are promising.

The communications of information among the vehicles to vehicles and vehicles to roadside provide a great opportunity for the development of new driver assistance systems. These systems will be able to disseminate and to gather real time information about other vehicles, road traffic with environmental conditions. Such data will be processed and analyzed to facilitate the driving by providing the user with useful information.

Intelligent Transportation Systems (ITS) includes the safety, security and efficiency of the transportation systems. It also enables new mobile applications and services for the travelling people. The field of intervehicular communications (IVC), including both vehicle-to-vehicle communications and vehicle-to 
roadside communications are recognized as an important component of Intelligent Transportation System in various national plans. Equipping vehicles with different types of on-board sensors, and V2V and V2R communication capabilities will allow large-scale sensing and decision / control actions in support of VANET objectives. Communication-based active safety is viewed as the next logical step towards proactive safety systems. These systems provide an extended information horizon to warn the driver or the vehicle of potentially dangerous situations at an early stage. In spite of the ongoing academic and industrial research efforts on VANETs, many research challenges remain. One of the interesting areas in VANET is Design and modification of Secure MAC layer for priority based and secured real time scalable access system. From the network perspective, security is one of the most significant challenges. Vehicle safety applications are among the major drivers for VANETs where people's lives are at stake, it is of course essential to secure VANETs against abuse. On the other hand, like all the other wireless networks, a MAC protocol should play a crucial role in scheduling application packet transmissions fairly and efficiently in VANETs, according to the quality of service (QoS) requirements of the applications with different message priorities for different types of applications to access DSRC (Dedicated Short Range [1][3].

Communications channels. The secure communication protocol is designed using time-stamp, digital signature, and trust certificate to guarantee the freshness of the message, message authentication and integrity, message non-repudiation, and privacy and anonymity of the senders. Vehicular Ad-Hoc Network (VANET) is an emerging new type of wireless networks in which vehicles constitute the mobile nodes in the network. This kind of networks supports vehicle-tovehicle and vehicle-to-gateway communications by providing a self-organized and multi-hop network. The
MAC Layer of IEEE 802.11 is simply based upon the carrier sense multiple access and collision avoidance access mechanism. There are many variations of MAC layers in 802.11 but, all are roughly same in functionalities. wireless medium is a lot different from the wired medium used in for example 802.3 (Ethernet), a new MAC layer needs to be defined to successfully mitigate problems such as interference, collisions and the increased security vulnerability. Carrier Sense Multiple Access Collision Avoidance (CSMA/CA) encounters problems such as interference, high collisions on overload and security vulnerability. Distributed coordination is another major problem in the access mechanism of the Vehicular ad hoc network. The basic rule for access under distributed coordination function (DCF) is 'if a station has to send a frame then first it senses the channel to see if somebody'. If the medium is busy, the station defers from sending and waits until the medium becomes available again. After the medium has become available, it waits for a predefined period of time, the DIFS or Distributed Inter-Frame Space. After this DIFS time the station enters a so-called contention window or backoff window. This is a time window divided in slots. The window size (in number of slots) depends on previous transmissions, but has a minimum and maximum defined by the standard. In 802.11 the contention window size is between 15 and 1023 slots and always increments in powers of 2 . The station then randomly chooses a number of slots (within the contention window), that is the number of time slots it will wait before it actually starts trying to transmit. This means that if two stations were both waiting for a transmission to end, they will not both start transmitting directly after the DIFS (and generate a collision). After picking a random number of slots a backoff timer starts counting down to zero, when it reaches zero the station can transmit. If, while waiting a random number of slots, the station senses another transmission, because another station was also waiting and picked a random number smaller than its own, it freezes the backoff timer [1]. 
After that the transmission and a DIFS of idle time on the medium, it restarts the backoff timer but does not pick a random number.

IEEE Std. 802.11 edition for information technologytelecommunication and information exchange between systems (Local and Metropolitan area network” under specific part -11 discussed the ad-hoc networking and its requirements. In this document technical and operational feasibility along with different technical standards for mobile ad-hoc networking are mentioned.

A Nasipuri, S.Ye, J. You, and R.E. Heromoto in their paper “A MAC Protocol for Mobile Ad-hoc Networks Using Directional Antennas” proposed a medium access control (MAC) protocol for an ad-hoc network of mobile and wireless terminals that are equipped with directional antennas. The use of directional antennas reduces the radio interference and inhances the packet throughput. The MAC protocol presented in this paper proposes a solution without help of any hardware.

J R. Yim, J. Guo, P. Orlik, and J. Zhang in their paper "Received Power-Based Prioritized Rebroadcasting for V2V Safety Message Dissemination” proposed Rapidly and robustly disseminating safety messages. This is an important goal for vehicle-tovehicle communications. They proposed receive powerbased prioritized rebroadcast scheme that minimizes the probability of packet collision during rebroadcast and maximizes the probability that a vehicle that is farthest away from the source rebroadcast an alert message first.

R. Jurdak, C. Videria Lopes, and P. Baldi, in their paper "A Survey, Classification and Comparative Analysis of Medium Access Control Protocols for Ad hoc Networks" proposed the Recent technological advances in wireless communications. This offer new opportunities and challenges for wireless ad-hoc networking. In the absence of the fixed infrastructure that characterizes traditional wireless networks, control and management of wireless ad-hoc networks must be distributed across the nodes, thus requiring carefully designed Medium Access Control (MAC) layer protocols. In this paper they survey, classify, and analyze 34 MAC layer protocols for wireless ad hoc networks, ranging from industry standards to research proposals. Through this analysis, 6 key features emerge: (1) channel separation and access; (2) topology; (3) power; (4) transmission initiation; (5) traffic load and scalability; and (6) range. These features allow them to characterize and classify the protocols, to analyze the tradeoffs produced by different design decisions, and to assess the suitability of various design combinations for ad hoc network applications. The classification and the tradeoff analysis yield design guidelines for future wireless ad hoc network MAC layer protocols.

R. K. Lam and P. R. Kumar, in their paper "Dynamic channel reservation to enhance channel access by exploiting structure of vehicular networks”, proposed VANET protocols need to exploit the special structure of vehicular networks. This structure includes the onedimensional nature of roads, the structure of lanes, the group mobility of vehicles, and the communication patterns of the envisaged applications. It is therefore of interest to examine how to specifically tailor VANET protocols to exploit all the above properties. In this paper, motivated by the goal of providing significantly better application level QoS, we study the MAC problem, and examine to what extent one can improve the performance of the mechanism employed in the IEEE 802.11p protocol. We design a dynamic channel reservation (DCR) protocol which leverages the special structure of VANET, and provides greater predictability in channel access, simplifying QoS provision. The key idea, in light of the periodic communication pattern of VANET applications, is to transform the per-packet channel contention mechanism of 802.11p into a pervehicle one in DCR. We implement the protocol on NS- 
2 for a comparative evaluation against 802.11p under realistic VANET scenarios. DCR demonstrates lower packet loss probability and higher throughput over 802.11p, and the simulation results appear promising enough to develop a complete protocol specifically for vehicular networks.

Ajay Chandra V. Gummalla and John O. Limb, in their paper "Wireless Medium Access Control Protocols”, proposed Technological advances, coupled with the flexibility and mobility of wireless systems, are the driving force behind the Anyone, Anywhere, Anytime paradigm of networking. At the same time, we see a convergence of the telephone, cable and data networks into a unified network that supports multimedia and real-time applications like voice and video in addition to data. Medium access control protocols define rules for orderly access to the shared medium and play a crucial role in the efficient and fair sharing of scarce wireless bandwidth. The nature of the wireless channel brings new issues like locationdependent carrier sensing, time varying channel and burst errors. Low power requirements and half duplex operation of the wireless systems add to the challenge. Wireless MAC protocols have been heavily researched and a plethora of protocols have been proposed. Protocols have been devised for different types of architectures, different applications and different media. This survey discusses the challenges in the design of wireless MAC protocols, classifies them based on architecture and mode of operation, and describes their relative performance and application domains in which they are best deployed

In this paper first we introduce the general terms related to VANET and Ad-hoc networking having technological requirements and feasibility. After that we discuss the issues related to different MAC layers such as IEEE 802.11, IEEE 802.11p (WAVE), Ad-hoc MAC and Directional Antenna Based MAC protocols functionalities. Finally, survey is taken on behalf of different performance factors. The result of survey on the basis of percentage scores for different performance factors are summarized in table 1 and in table 2.

\section{MAC Issues}

Medium access control sub layer design is a measure issue related for the vehicular ad hoc network. Basically, MAC layer in local area network either in wired medium or wireless is broadcast in nature. In a broadcast network that can access the channel is governed by the access mechanism and protocols. For general applications of wireless network the Carrier Sense Multiple Access is suitable and working very well but in case of VANET this CSMA is not suitable due to many reasons. The reasons are mobility and switching, more collision on load increase, Critical and life saving safety information dissemination, quickness, accuracy, privacy and confidentiality. These requirements are the basic need in the Vehicular ad hoc network. The critical information like one hop safety message requires quick and efficient dissemination to the vehicles those are near to vehicle. If this is not performed then vehicle crash may occur. Aloha and S-Aloha are not suitable because low frame success rate. Frequency division multiple access has a problem on the time of switching from one cell to other cell on roadside. Time division multiple access is also not efficient for the VANET MAC layer due to dynamic mobility of vehicles on roadside. On combining two different multiple channel access protocol produces delay in processing that is not allowed in the vehicle to vehicle communication. If this is allowed then active safety message will reach to the vehicle which are one hop distant from the originator vehicle later or after crash. Due to mentioned facts the MAC layers such as IEEE 802.11, IEEE 802.11p (WAVE), Ad-hoc MAC and Directional Antenna based MAC are not suitable VANET application scenarios. Probable Scenario of Vehicle to Vehicle and Vehicle to Roadside Communication is given in figure 1. 


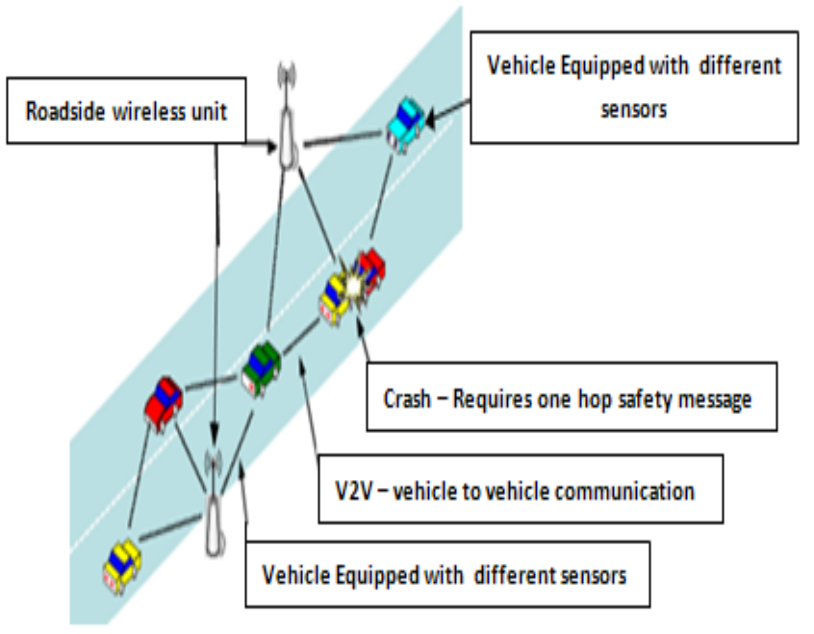

Figure 1: V2V and V2R scenarios

Figure 1 shows there are many vehicles on the roadside. The roadside unit disseminates and gathers the information in V2R communications and in V2V communications the vehicles disseminates and gathers the information from the vehicles. The MAC layer plays important roles in this type of ad hoc networks. The MAC layer in $\mathrm{V} 2 \mathrm{~V}$ and $\mathrm{V} 2 \mathrm{R}$ should have to be compatible with each other to flexible communication between vehicles to vehicles and vehicles to roadside communications.

\subsection{IEEE 802.11 MAC}

In this MAC the Inter Frame Space(IFS) is very important and Sort Inter frame Space(SIFS) should be greater than Distributed Inter Frame Space(DIFS). [4][5][6]. The design of MAC is given in picture 2.

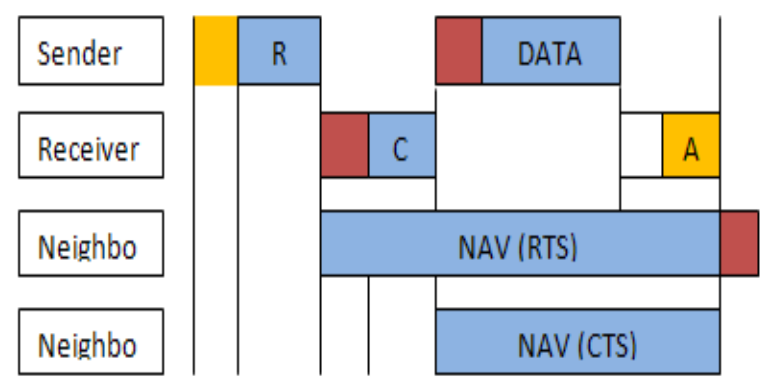

Figure 2: Working of IEEE 802.11 MAC Layer

\subsection{IEEE 802.11p WAVE}

WAVE (Wireless Access in Vehicular Enviournment) is an amendment to all IEEE 802.11 protocols and adapted for the IEEE 802.11 standard for inter-vehicular communications to provide low latency and high reliability. It was published in November 2010 according to the official IEEE 802.11 working group project timelines. 802.11p (WAVE in detail) will be used as the groundwork for Dedicated Short Range Communications (DSRC), it is architecture standard looking at vehiclebased communication networks, particularly for applications such as toll collection, vehicle safety services, and electronic commerce transactions via vehicles. The ultimate vision is a nationwide network in United States(US) that enables communications between vehicles and roadside access points or vehicles to other vehicles.[7][8]. Working diagram for IEEE 802.11p is given in figure 3 .

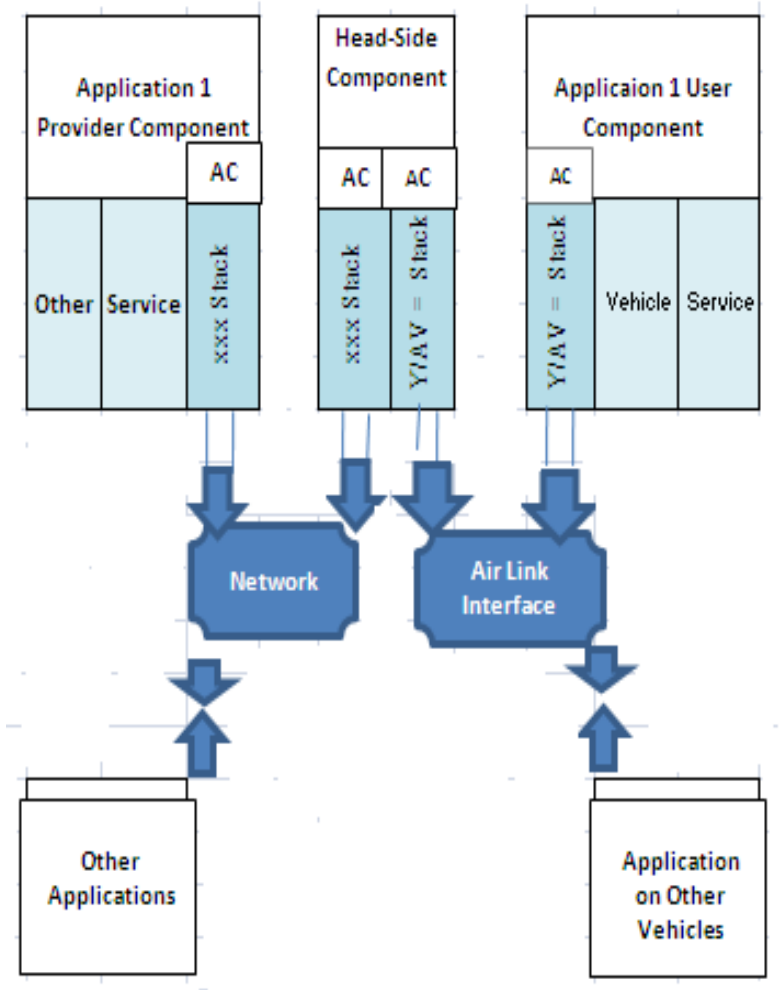

Figure: 3 IEEE 802.p WAVE Working diagram 


\subsection{Adhoc MAC}

This Ad-hoc MAC is based upon the circuit switching method. The sender vehicle has to establish the circuit first and then frame is being transmitted either to the vehicles or to roadside station.[9][10]. Here the Reliable Reservation Aloha (RR- ALOHA) protocol is used. The medium is divided into several repeated time frames and each frame again divided into n numbers of time slots. The functional diagram of this Ad-hoc MAC is given in figure 4 .

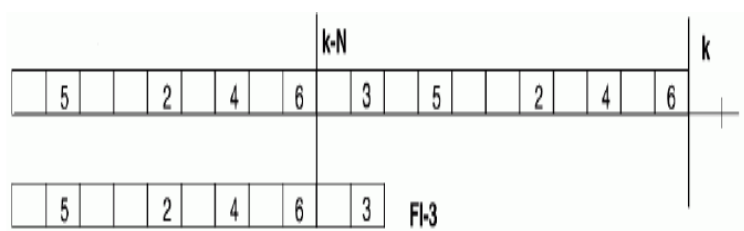

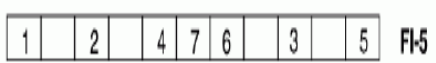

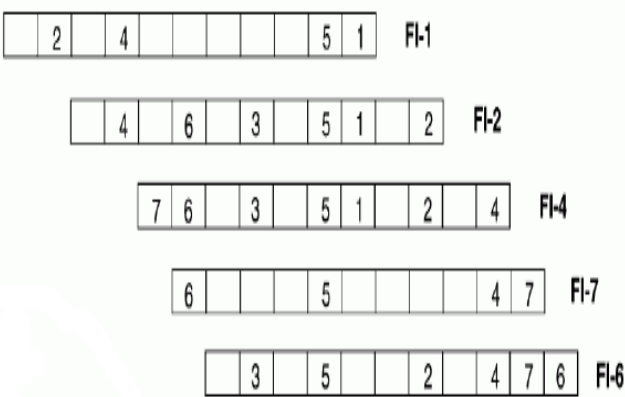

Figure: 4 Functional behavior of Ad-hoc MAC.

\subsection{Directional Antenna Based MAC}

Directional antenna based MAC increase the area of coverage and hence it has greater channel capacity. It is good solution for vehicular network because a vehicle can only run on the road.[2][3]. The working scenario of directional antenna based MAC is given in figure 5 .

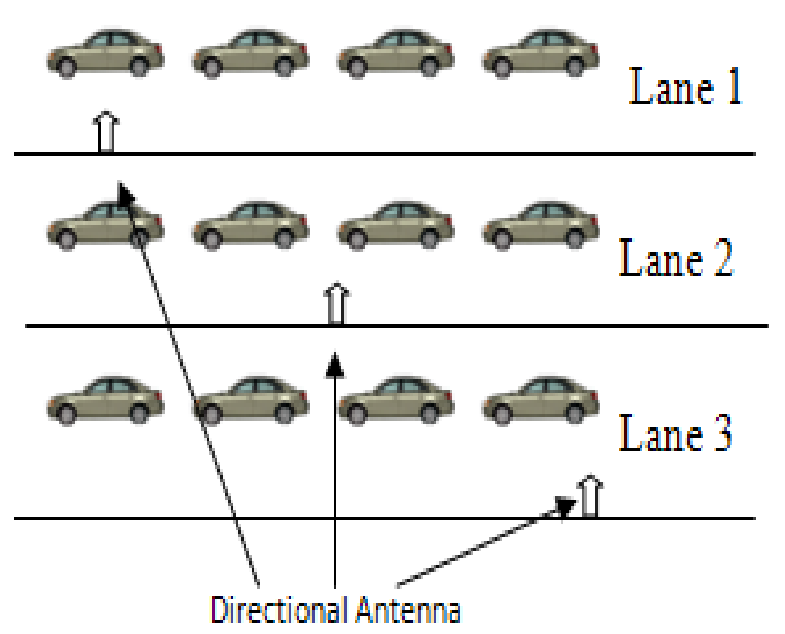

Figure 5: Directional Antenna based communication in VANET

Directional MAC is protocol used at MAC Layer and it uses 4 way handshaking method of IEEE 802.11. Each vehicle is equipped with GPS system which can determine its geographical position [11][12]. The handshaking scenario is illustrated in figure 6.

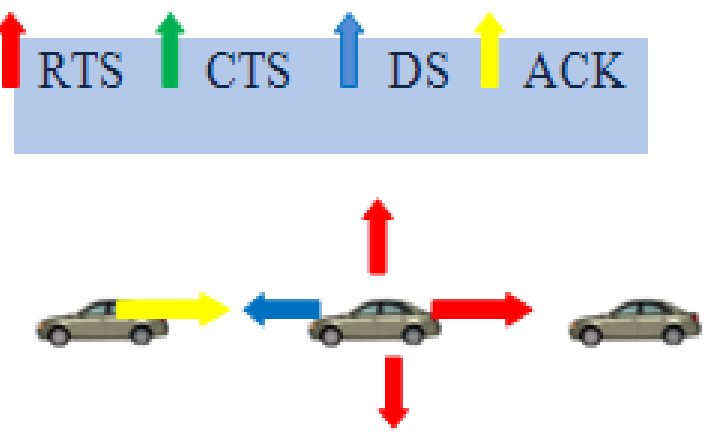

Figure 6: 4 Way Handshaking

\section{Performance Evaluation for Different MAC of VANET}

Performance evaluation of different MAC protocols is based upon the many parameters. The different parameters for performance evaluation are mobility, accuracy, privacy and confidentiality, safety control priority and delay control. The mobility factor is one of the major factors in VANET application. This includes switching of vehicle device one cell to other cell and continuation of information exchanges between vehicles 
to vehicles and vehicles to roadside units. Accuracy determines how much accurate the information received. Privacy and confidentiality is maintained for the different electronic commerce applications. The VANET is public network hence privacy should have to be provided to individual user. The priority for safety control critical message is very important for the prevention of the crash and accident. This performance factor should be considered as the main factor in the vehicular network scenario. Finally, suitability of different manufacturer hardware such as antenna, GPS systems, computing platform and software such as networking components on different layers at protocol suit should be interoperable These all parameters are categorized under low, medium and high with given condition. The condition makes it more specific in nature for a specified MAC layer protocol in VANET application area. Table 1 . shows the different performance for MAC protocols. Each performance factor has $100 \%$ Score with Least, low, medium and high includes 5\%, 10\%, 15\% and 20\% respectively. These performance factors are analyzed on behalf of the current usability and with conditional suitability for the VANET. The percentage score of different performance factors are obtained by the analysis of the individual performance factor in ad-hoc enviournment. These all performance factors with different protocols are summarized under Table 1.

\begin{tabular}{|l|l|l|l|l|}
\hline \multicolumn{5}{|c|}{ Table 1. Performance of different MAC Layer Protocols for } \\
VANET \\
\hline $\begin{array}{l}\text { Parameters } \\
\text { Factors }\end{array}$ & $\begin{array}{l}\text { IEEE } \\
\mathbf{8 0 2 . 1 1}\end{array}$ & $\begin{array}{l}\text { IEEE } \\
\mathbf{8 0 2 . 1 1 p} \\
\text { WAVE }\end{array}$ & $\begin{array}{l}\text { Ad-hoc } \\
\text { MAC }\end{array}$ & $\begin{array}{l}\text { Directional } \\
\text { Antenna } \\
\text { based } \\
\text { MAC }\end{array}$ \\
\hline $\begin{array}{l}\text { Mobility } \\
\text { Accuracy }\end{array}$ & Least & Medium & Medium & Least \\
\hline $\begin{array}{l}\text { Privacy \& } \\
\text { Confidentiality }\end{array}$ & Least & Mow & Low & High \\
\hline $\begin{array}{l}\text { Safety critical } \\
\text { message } \\
\text { priority }\end{array}$ & Least & Low & Least & Least \\
\hline $\begin{array}{l}\text { Delay control } \\
\text { Suitability }\end{array}$ & Least & Medium & Low & Medium \\
\hline
\end{tabular}

The given information of performance parameters are survey based for the MAC Layers of different protocols. The final performance of MAC Layer protocols are summarized in table 2 . which is based upon the table 1 .

\begin{tabular}{|c|c|c|}
\hline \multicolumn{3}{|c|}{$\begin{array}{r}\text { Table 2. Performance Factor in } \% \text { fo } \\
\text { Protocols }\end{array}$} \\
\hline MAC Layer Protocol & $\begin{array}{l}\text { Performance Factor } \\
\text { MAC Layer of VAN }\end{array}$ & $\begin{array}{l}\text { (\%) of } \\
\text { ET }\end{array}$ \\
\hline IEEE 802.11 & $5+10+5+5+5+5$ & $=35 \%$ \\
\hline IEEE 802.11p WAVE & $15+15+10+10+15+15$ & $=\mathbf{8 0} \%$ \\
\hline Ad-hoc MAC & $15+5+10+5+10+5$ & $=45 \%$ \\
\hline $\begin{array}{l}\text { Directional Antenna } \\
\text { Based MAC }\end{array}$ & $5+20+20+5+15+5$ & $=70 \%$ \\
\hline
\end{tabular}

Table 2 shows that IEEE 802.11 MAC layer is least suitable MAC layer for VANET and Directional Antenna based MAC layer is highly suitable in case of VANET. 


\section{Conclusion}

In this paper we have proposed the survey of MAC layer of different protocols. The feasibility of VANET on current existing MAC layers of different protocols under wireless medium are studied. According to study of the MAC layers of different protocols in which IEEE 802.11p (WAVE) is the most suitable protocol and IEEE 802.11 is the least suitable protocol for the VANET. The requirement at the level of MAC layer in VANET is not fulfilled by the score of $80 \%$. To cover up the requirement for crash and other scenarios of VANET a MAC layer performance factor should be $95 \%$ and above. Hence, none of the studied protocols is suitable according to the requirement of the Vehicular Ad-hoc Network. So, it is required to discover the MAC layer protocol that would have 95\% and above performance factor by table 1 . and table 2 .

\section{References}

[1] "IEEE Std 802.11 Edition, "Information Technology - Telecommunication and information exchange between systems - Local and metropolitan area network” - Specific requirement part - 11: Wireless Lan Medium Access Control (MAC) and Physical Layer (PHY Specifications).

[2] A. Nasipuri et al., _A MAC Protocol for Mobile Ad Hoc Networks Using Directional Antennas, Proceedings of IEEE WCNC 2000, vol. 1, pp. 12141219, September 2000.

[3] Z. Huang et al., _A Busy Tone-Based Directional MAC Protocol for Ad Hoc Networks,_ Proceedings of IEEE MILCOM 2002, October 2002.

[4] Ajay Chandra V. Gummalla and John O. Limb,_Wireless Medium Access Control Protocols,_ IEEE Commun. Surv., 2000.

[5] T. Korakis, G. Jakllari and L. Tassiulas, _A MAC protocol for full exploitation of Directional Antennas in Ad-hoc Wireless Networks, _ Proceedings of 4th ACM Int. Symp. on Mobile Ad Hoc Networking and computing, Annapolis, Maryland, June 2003.

[6] R. Jurdak, C. Videria Lopes, and P. Baldi,_A Survey, Classification and Comparative Analysis of Medium Access Control Protocols for Ad hoc Networks, _ IEEE Commun. Surv., 2004.

[7] "IEEE 1609 - Family of Standards for Wireless Access in Vehicular Environments (WAVE)," U.S. Department of Transportation, January 9, 2006.
[8] Fei Ye, Raymond Yim, Jianlin Guo, Jinyun Zhang and Sumit Roy, "Prioritized Broadcast Contention Control in VANET", Mitsubishi Elect. Research Labs, Cambridge, MA 02139 and University of Washington, Seattle, WA 98195.

[9] V. S. Communications, "Vehicle safety communications project-final report," tech. rep., April 2006.

[10] IEEE, IEEE 1609.4-Standard for Wireless Access in Vehicular Environments (WAVE) - Multi-channel Operation.

[11] R. Yim, J. Guo, P. Orlik, and J. Zhang, "Received Power-Based Prioritized Rebroadcasting for V2V Safety Message Dissemination,” in Proc. of Int. Transport. Sys. World Congr., Sep. 2009.

[12] R. K. Lam and P. R. Kumar. Dynamic channel reservation to enhance channel access by exploiting structure of vehicular networks. In VTC 2010-Spring: Proceedings of the IEEE $71^{\text {st }}$ Vehicular Technology Conference, Taipei, Taiwan, 2010.

Vimal Bibhu : Master in Technology in Computer Science and Engineering and pursuing Doctor of Philosophy in Computer Science from BRA Bihar University, Mujaffarpur, Bihar, India. He has published many research papers in different international journals. He is also member of SERC, ISCSIT, IEANG.

Dr. Dhirendra Kumar Singh : Doctor of Philosopphy in Mathematics and currently working on the post of Professor under University Department of Mathematics, BRA Bihar University, Mujaffarpur, Bihar, India. 